\title{
Activation of the ERK1/2 pathway by the CaMEK gene via adeno-associated virus serotype 9 in cardiomyocytes
}

\author{
Y.-N. Yang ${ }^{1,2 *}$, W.-N. Ji ${ }^{1,2 *}$, Y.-T. Ma ${ }^{1,2}$, X.-M. Li ${ }^{1,2}$, B.-D. Chen ${ }^{2}$, Y. Xiang ${ }^{1}$ \\ and F. Liu ${ }^{2}$ \\ ${ }^{1}$ Department of Cardiology, \\ First Affiliated Hospital of Xinjiang Medical University, Urumqi, P.R. China \\ ${ }^{2}$ Xinjiang Key Laboratory of Cardiovascular Disease Research, \\ Urumqi, P.R. China \\ *These authors contributed equally to this study. \\ Corresponding author: Y.-T. Ma \\ E-mail: myt-xj@163.com / myt_xj@sina.com
}

Genet. Mol. Res. 11 (4): 4672-4681 (2012)

Received January 6, 2012

Accepted June 1, 2012

Published October 17, 2012

DOI http://dx.doi.org/10.4238/2012.October.17.1

\begin{abstract}
Extracellular signal-regulated kinase (ERK1/2) is one of the mitogen-activated protein kinases, key components of the reperfusion injury salvage kinase pathway, which plays an important role in protecting the myocardium from lethal ischemia-reperfusion injury. Constitutive activation of the mitogen-activated protein kinase kinase 1 (CaMEK) can promote ERK1/2 expression, which is thereby expected to exert protective action on the heart against ischemia-reperfusion injury. The adeno-associated virus serotype 9 vector (AVV9) is a novel tool for gene therapies targeting human diseases owing to its nonpathogenic capability for transducing nondividing cells and its long-term transgene expression. We used a recombinant AAV9 vector to deliver the CaMEK gene into cardiomyocytes and assessed whether AAV9 vector-mediated CaMEK gene transfection could enhance the long-term expression and activity of ERK1/2. Our observations suggest that AAV9-mediated gene expression is preferentially restricted
\end{abstract}


to cardiomyocytes and that mediated CaMEK gene transfection enhanced the expression of ERK $1 / 2$ phosphorylation and consequently upregulated the expression of downstream components of ERK1/2 and its transcription factors.

Key words: Recombinant adeno-associated virus serotype 9; Constitutive activation of the mitogen-activated protein kinase kinase 1; Extracellular signal-regulated kinase

\section{INTRODUCTION}

Ischemic heart disease is the leading cause of death in the developed world. Reperfusion therapy is the most effective treatment for ischemic heart disease, but it causes reperfusion injury at the same time it saves surviving myocardial cells, directly affecting the prognosis of patients. Previously studies have shown that the reperfusion injury salvage kinase pathway plays an important role in salvaging viable myocardium, limiting infarct size, and protecting the myocardium from lethal ischemia-reperfusion (I/R) injury (Hausenloy et al., 2005). Extracellular signal-regulated kinase (ERK1/2), the key component of the reperfusion injury salvage kinase pathway, is associated with cell survival and proliferation (Cho et al., 2009). Previous studies have proven that the ERK $1 / 2$ signaling pathway plays a central role in regulating cardiomyocyte $(\mathrm{CM})$ viability after an apoptotic insult on cultured CMs (Bueno and Molkentin, 2002). Furthermore, a heart I/R model has demonstrated that the activation of ERK1/2 reduces I/R injury-induced apoptosis ( $\mathrm{Li}$ et al., 2006). Enhancement of ERK1/2 activity is expected to reduce myocardial $\mathrm{I} / \mathrm{R}$ injury and improve prognosis in patients with acute myocardial infarction.

Notably, mitogen-activated protein kinase kinase 1 (MEK1) is the major upstream activator of ERK1/2 (Lips et al., 2004), and within the heart, the MEK1-ERK1/2 signaling is thought to be cardioprotective through direct antagonization of myocyte apoptosis (Bueno and Molkentin, 2002). Recently, transfection of constitutive activation of the mitogen-activated protein kinase kinase 1 (CaMEK) into cells has been reported to promote ERK1/2 expression; activated ERKs then translocate into the nucleus, where they activate nuclear transcription factors such as Elk-1, c-fos, and c-jun, thereby regulating cell proliferation and differentiation (Treisman, 1996; Manning et al., 2002). Such proliferative effects of CaMEK in confluent cells of an intestinal epithelial line (IEC-6) have been reported, and the silencing of MEK expression significantly reduces the extent of ERK1/2 phosphorylation, inhibiting cell proliferation (Voisin et al., 2008). MEK1 transgenic mice have also been shown to be resistant to I/R injury and have significantly reduced myocardial infarct size (Lips et al., 2004). However, the above methods such as silencing of MEK expression and MEK1 transgenic mice interfere with ERK1/2 signaling pathways elsewhere in the body, because full activation of the ERK1/2 signaling pathway causes excessive activation and proliferation in the cells of other organs, inducing effects such as tumors (Kuno et al., 1998; Licato and Brenner, 1998; Lemieux et al., 2009). Therefore, safer and more effective methods of myocardium-targeted transduction must be found that do not affect the normal function of other tissues and organs.

The use of adeno-associated virus (AAV) vectors has emerged as a novel method for gene therapy targeting human diseases owing to the nonpathogenic capability of these vectors 
for transducing nondividing cells and long-term transgene expression (Prasad et al., 2011). AAV serotype 9 vectors (AVV9) are of particular interest owing to their high efficiency of gene transfection in the heart (White et al., 2011). In the present study, we used a recombinant AAV9 (rAAV9) vector to deliver the CaMEK gene into CMs and assessed whether AAV9 vector-mediated CaMEK gene transfection could enhance long-term ERK1/2 expression and activity. The results of the study are intended to provide an experimental basis for gene therapy in promoting cell survival and reducing $\mathrm{I} / \mathrm{R}$ injury.

\section{MATERIAL AND METHODS}

\section{Plasmids and vector construction}

Vectors of AAV9-CBA-eGFP (enhanced green fluorescent protein) and AAV9-CBAeGFP-MEK-S217E-S221E were purchased from Virovek (USA).

\section{Primary CM culture}

Primary CMs were prepared as described elsewhere (Simpson and Savion, 1982), with some modifications. Briefly, neonatal rat ventricular CMs from 1- to 3-day-old Sprague Dawley rats were digested using $0.025 \%$ trypsin (Ameresco, USA) and maintained in highglucose Dulbecco's modified Eagle's medium (DMEM; HyClone, USA) supplemented with $20 \%$ fetal bovine serum (Gibco, USA), $100 \mathrm{mg} / \mathrm{mL}$ ampicillin, and $0.1 \mathrm{mg} / \mathrm{mL}$ streptomycin. The cells were cultured at $37^{\circ} \mathrm{C}$ in a humidified atmosphere containing $5 \% \mathrm{CO}_{2}$ for $2 \mathrm{~h}$. The non-adherent cells were CMs, which were harvested and seeded into another dish at a density of $1 \times 10^{5}$ cells. Bromodeoxyuridine $(10 \mu \mathrm{M}$; Sigma-Aldrich, USA) was added to inhibit the proliferation of non-CMs (NCM). Adherent cells were NCMs, mainly fibroblasts. After plating for $48 \mathrm{~h}$, the media were replaced with fresh medium containing the components specified above. This protocol yielded more than $98 \%$ CMs as defined by NKX2.5 antibody (1:100 dilutions; Santa Cruz, USA). CMs were maintained for $72 \mathrm{~h}$ before treatment and NCMs were harvested and seeded at a density of $1 \times 10^{5}$ cells/dish for the research of AAV9 CMs specifically. After transfection with AAV9-eGFP, the expression of eGFP in the CMs and NCMs was examined daily using an inverted fluorescence microscope (LEICA-DMI4000B, Germany) for 9 days. Cells were harvested on the 5th day (the peak time of the expression of AAV9 selected from preliminary experiments) to examine the expression of eGFP protein with Western blot.

\section{Experimental protocols (grouping)}

After $72 \mathrm{~h}$ of culture, the CMs were subjected to transfection of AAV9 and AAV9 + CaMEK. The multiplicities of infection (MOI) of AAV9 and AAV9 + CaMEK were selected from preliminary experiments $\left(\mathrm{MOI}=6 \times 10^{5} \mathrm{vg} /\right.$ cell) demonstrating transfection efficiency of more than $90 \%$ at the peak time. The cells were divided into groups as follows:

(1) Control: normal cells without transfection $(\mathrm{MOI}=0)$.

(2) rAAV9-eGFP transfected: the medium was discarded, and the cells were washed twice with serum-free DMEM. AAV9 was added to serum-free DMEM to cover the cell 
surface. MOI was $6 \times 10^{5} \mathrm{vg} / \mathrm{cell}$. After $2 \mathrm{~h}$ of incubation at $37^{\circ} \mathrm{C}$ and $5 \% \mathrm{CO}_{2}$, the virus solution was kept and serum-containing DMEM was added to a final concentration of fetal bovine serum of $20 \%$. Cells were maintained at $37^{\circ} \mathrm{C}$ and $5 \% \mathrm{CO}_{2}$ before collection at the peak time of AAV9 expression (the peak time at the 5th day was selected from preliminary experiments).

(3) rAAV9-eGFP-CaMEK transfected group: transfection methods and procedures followed those described in (2) except that rAAV9-eGFP-CaMEK replaced rAAV9-eGFP.

(4) rAAV9-eGFP transfected + PD98059 treatment: transfection methods and procedures followed those described above. Pretreatment with PD98059 (an MEK1/2 inhibitor, 20-50 $\mu \mathrm{M}$; Invitrogen, USA) took place $30 \mathrm{~min}$ before the administration of rAAV9-eGFP. The dose of PD98059 was determined from preliminary experiments demonstrating inhibition of ERK1/2 activation.

(5) rAAV9-eGFP-CaMEK transfected + PD98059 treatment: transfection methods and procedures followed those described in (4), except that rAAV9-eGFP-CaMEK replaced rAAV9-eGFP.

\section{Fluorescence microscopy}

To examine the efficacy of gene transfection by the rAAV9 vector, we observed eGFP expression every day with an inverted fluorescence microscopy (LEICA-DMI4000B) for 9 days.

\section{Total protein extraction}

Cells were collected and washed with phosphate-buffered saline and lysed with a lysis buffer. The cell lysates were sonicated for 2 min to break down the unlysed cells further. The protein concentrations were quantified using a Bio-Teke protein assay kit (Bio-Teke, China).

\section{Western blot analysis}

Western blot analysis was carried out as described elsewhere (Li et al., 2009). Antibodies were used from the following sources: p70S6K (Thr389), Phospho-p44/42 MAPK (ERK1/2) (Thr202/Tyr204), p44/42 MAPK (ERK1/2), MEK1/2, c-Fos, c-Jun, Elk-1, eGFP, glyceraldehyde 3-phosphate dehydrogenase (all from Cell Signaling Technology, USA), and NKX2.5 (Santa Cruz).

\section{Immunofluorescence analysis}

Cells were prepared as described elsewhere (Lemieux et al., 2009). Primary antibody [total (t-ERK) and phosphorylated (p-ERK)] incubation was carried out (1:100 dilutions) overnight at $4^{\circ} \mathrm{C}$ overnight. The cells were then washed 3 times with phosphate-buffered saline and incubated with DyLight 594 AffiniPure goat anti-rabbit immunoglobulin G-labeled secondary antibodies (1:100 dilutions; EaythOx, LLC, USA) for $30 \mathrm{~min}$ at room temperature. They were observed using fluorescence microscopy after incubation with 4',6-diamidino-2-phenylindole staining solution $(20 \mathrm{mg} / \mathrm{mL}$; Roche, Germany) for $15 \mathrm{~min}$. Negative controls (no primary antibody) were included in all experiments. Experiments were repeated at least 3 times. 


\section{Statistical analysis}

One-way analysis of variance was used when multiple groups were compared. All results are reported as means \pm standard error. A P value of $<0.05$ was considered to be statistically significant.

\section{RESULTS}

\section{AAV9 mediating long-term and CM-specific gene expression}

We examined the cell type efficiency and specificity of eGFP expression and found that AAV9-mediated eGFP expression observed on fluorescence microscopy was abundant in CMs but almost undetectable in NCMs (mainly fibroblasts; Figure 1A). Western blot revealed that the expression of eGFP in CMs was dramatically higher than that in NCMs on the 5th day, which was the peak time of eGFP expression (Figure 1B), indicating that AAV9-mediated gene expression was preferentially restricted to CMs.

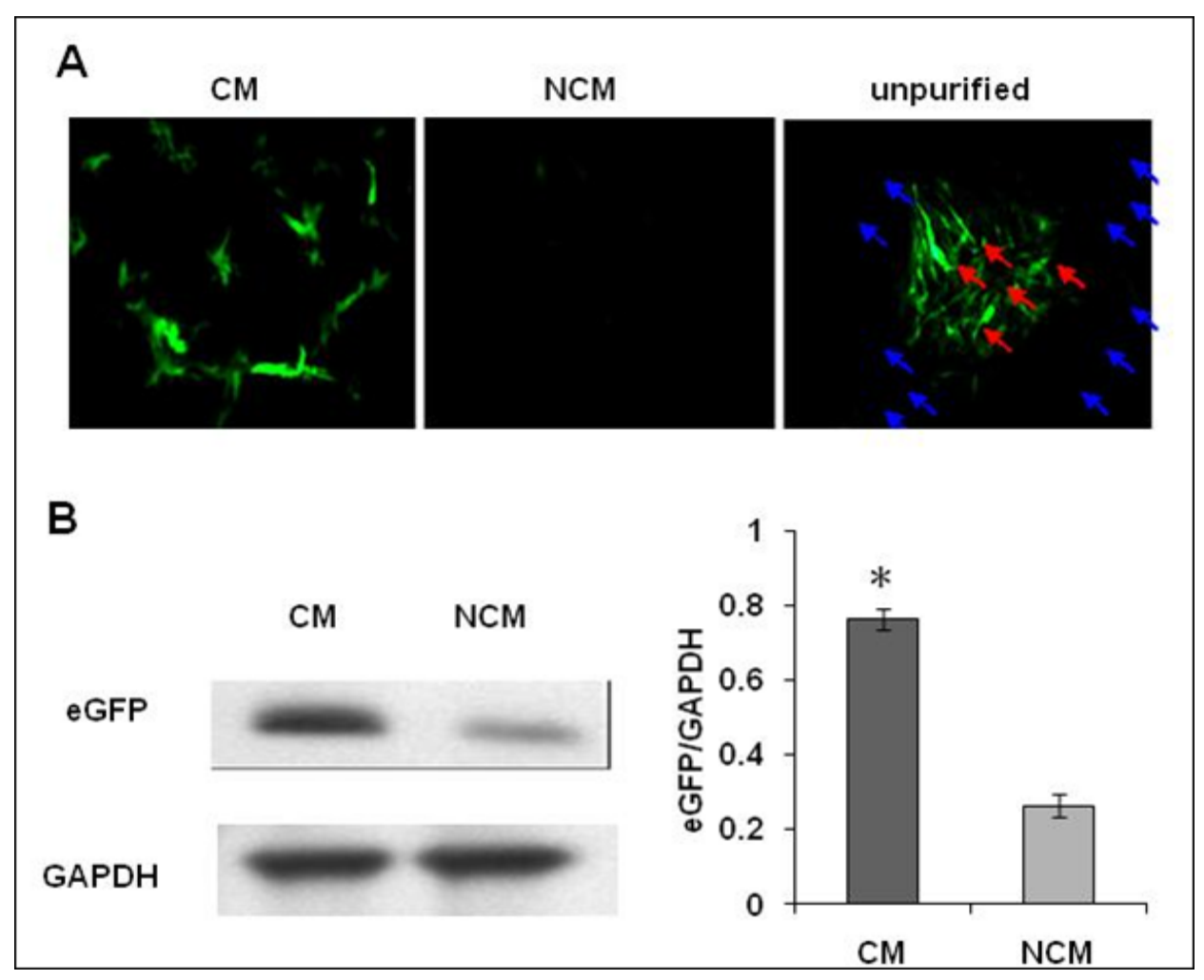

Figure 1. A. eGFP expression observed by fluorescence microscopy was abundant in cardiomyocyte (CM), but almost undetectable in non-CM (NCM), unpurified cells (including $\mathrm{CM}$ and $\mathrm{NCM}$ ) indicating the same results $(\rightarrow \mathrm{CM} ; \rightarrow \mathrm{NCM})$. B. Western blot analysis of eGFP protein expression between $\mathrm{CM}$ and NCM cells. ${ }^{*} \mathrm{P}<0.05$ vs NCM. 
The duration of eGFP expression in CMs lasted for 9 days after transfection and peaked on the 5 th day. These findings were confirmed with both inverted fluorescence microscopic observation and protein quantitation (Western blot; Figure 2).

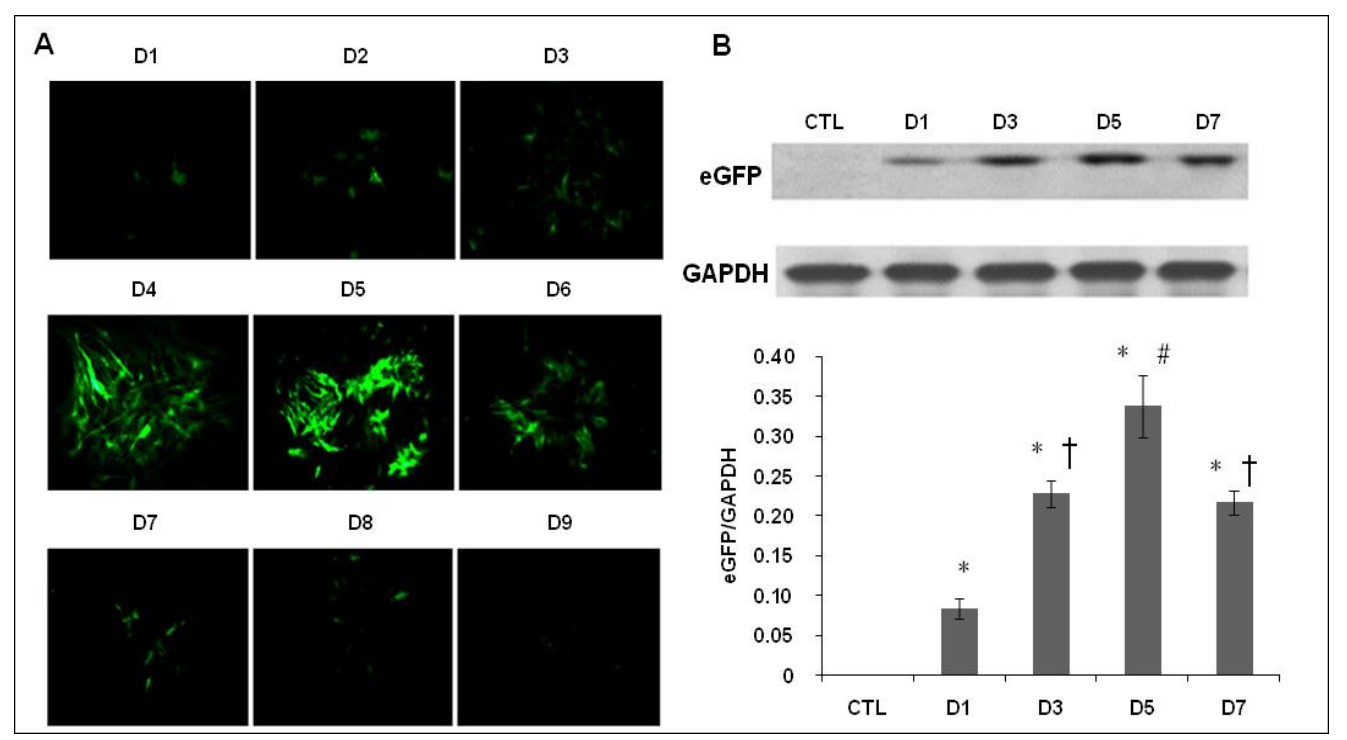

Figure 2. A. eGFP expression was observed by inverted fluorescence microscopy. The peak level of eGFP expression was at the fifth day, and was lasted for 9 days (D1-D9) after transfection. B. Expression of GFP protein was quantifield by Western blot. $\mathrm{CTL}=$ control. ${ }^{*} \mathrm{P}<0.05$ vs $\mathrm{CTL} ;+\mathrm{P}<0.05$ vs $\mathrm{D} 1, \mathrm{D} 5$; ${ }^{\#} \mathrm{P}<0.05$ vs $\mathrm{D} 3, \mathrm{D} 5$.

\section{rAAV9-mediated transfection of CaMEK and of ERK1/2 pathway activation}

After confirming cardiomyocyte-specific gene expression mediated by rAVV9 transfection, we transfected CMs with AAV9-eGFP-CaMEK and performed Western blot analysis to examine the expression levels of MEK and p-ERK1/2 and its downstream component P70S6 kinase (P70S6K). Five days after transfection, the expression levels of MEK, p-ERK, and P70S6K were significantly increased (by 45.3, 24.2, and 35\%, respectively) compared with those in the untreated control group, indicating an activation of the ERK $1 / 2$ pathway mediated by CaMEK transfection. The timing for reaching peak expression levels of MEK, p-ERK1/2, and P70S6K was consistent with that for rAAV9 vector-mediated eGFP expression. However, such enhanced expression of MEK, p-ERK1/2, and P70S6K was completely abolished by treatment with a selective MEK1 inhibitor, PD98059 (Figure 3A). Interestingly, even in empty rAAV9 vector-transfected CMs, PD98059 treatment also significantly suppressed expression of MEK, p-ERK1/2, and P70S6K. No significant difference was found in the expression of t-ERK1/2 among the 5 groups, indicating that rAAV9 vector-mediated CaMEK transfection or treatment with the MEK1 inhibitor had no effect on $\mathrm{t}$-ERK1/2 protein expression. 
Furthermore, immunofluorescence revealed that in rAAV9-CaMEK-transfected CMs, t-ERK1/2 was located in the cytoplasm and p-ERK1/2 was almost entirely located in the nucleus, whereas in the control group, t-ERK1/2 was located in the cytoplasm, consistent with rAAV9-CaMEK-transfected CMs, but only a small portion of p-ERK1/2 appeared in the nucleus (Figure 3B).

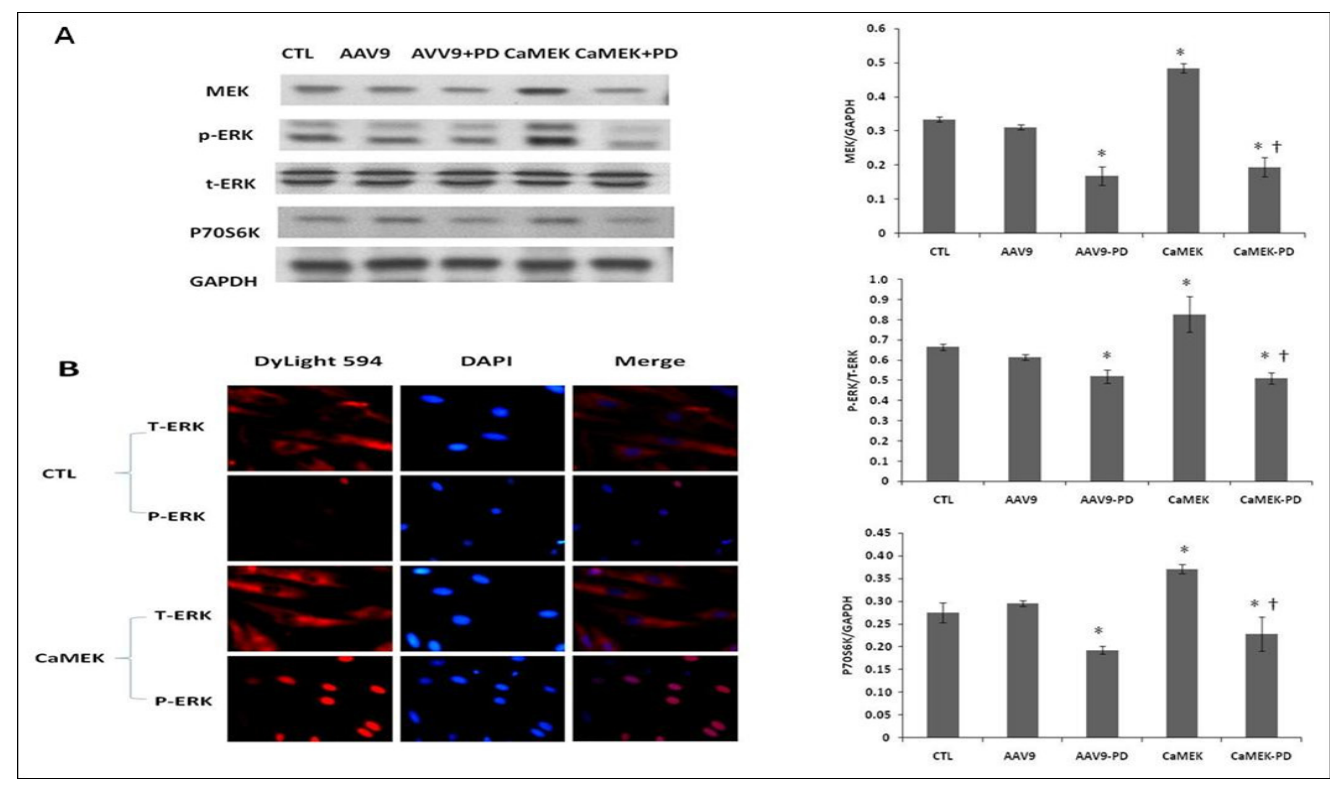

Figure 3. Analysis of protein expression of MEK, p-ERK and P70S6K in different groups [control (CTL), AAV9, AAV9 + PD98059 (PD), CaMEK, CaMEK + PD98059 (PD)] by Western blot. A. The amount of MEK p-ERK and P70S6K significantly increased (by 45.3, 24.2 and 35\%, respectively) in the CaMEK-transfected group. After administration of PD98059, the amount of MEK p-ERK and P70S6K attenuated significantly ( ${ }^{*} \mathrm{P}<0.05$ vs CTL, AVV9; $+\mathrm{P}<0.05$ vs CaMEK). B. Position of $\mathrm{p}-\mathrm{ERK}$ detected by immunofluorescence. In rAAV9-CaMEK-transfected cardiomyocyte, t-ERK1/2 was located in the cytoplasm, p-ERK1/2 was almost all located in the nucleus, whereas, in the CTL group, the t-ERK1/2 was located in the cytoplasm consistent with rAAV9-CaMEK-transfected cardiomyocyte, but there was only a small part of p-ERK $1 / 2$ located in the nucleus.

\section{Expression of transcription factors activated by rAAV9-mediated transfection of CaMEK}

We next examined whether activation of ERK1/2 by rAAV9-CaMEK gene transfection would activate nuclear transcription factors including Elk-1, c-fos, and c-jun, known downstream targets of the ERK1/2 pathway. Immunoblotting showed that the expression levels of Elk-1 and c-fos, but not c-jun, in the nucleus were significantly increased compared with those in controls, and inhibition of MEK by PD98059 attenuated the upregulation of Elk-1 and c-fos (Figure 4). 


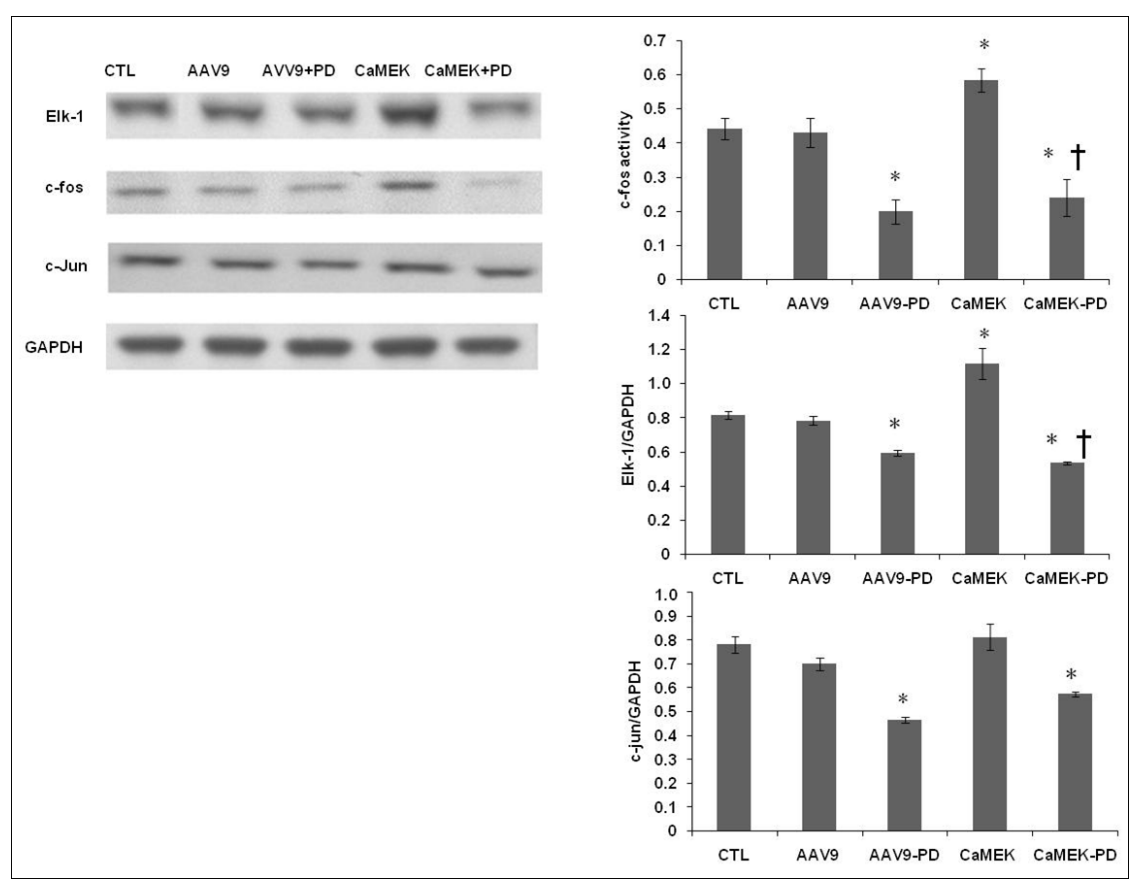

Figure 4. Expression of nuclear transcription factor after transfection with AAV9-CaMEK. Expression of Elk-1 and c-fos in the nucleus was significantly increased compared to their corresponding control levels, but not c-jun, and inhibition of MEK by PD98059 attenuated the upregulation of Elk-1 and c-fos ( $* \mathrm{P}<0.05$ vs control (CTL), AVV9; $+\mathrm{P}<0.05$ vs CaMEK).

\section{DISCUSSION}

In this study, we assessed the efficacy and specificity of AAV9-mediated gene transfection in cultured primary CMs and the effects of CaMEK gene transfer on the activation of the ERK1/2 pathway and protection against I/R injury. We made several findings: 1) AAV9mediated gene expression was preferentially restricted to CMs; 2) AAV9 vector-mediated CaMEK gene transfection resulted in enhanced expression of MEK and p-ERK1/2, and 3) CaMEK gene transfection upregulated the expression of downstream components of ERK1/2 and its transcription factors.

AAV vector-mediated gene transfer has emerged as a novel method for the treatment of human disease because of its effective and stable transduction in target organs. AAV9, a new serotype of AAV, is considered an ideal vector for cardiac gene therapy because studies have shown that it is best suited for gene delivery to the heart (Hajjar and Samulski, 2006; Pacak et al., 2006). Nevertheless, most studies of CM-specific AAV9 were in vivo studies. Herein, we demonstrate that AAV9 provides superior direct gene transfer in CMs in vitro. The rAAV9 vector provided robust and early onset gene expression in CMs, and the eGFP expression increased rapidly over the entire experimental period. Compared to myocardial cells, NCM cells displayed a prolonged lag phase and low levels of gene expression after rAAV9 vector administration. 
Notably, ERK1/2 is one of the mitogen-activated protein kinases. Studies of cultured systems and transgenic and gene-targeted mice have shown that it plays an important role in the heart. Activation of the ERK1/2 pathway has recently been shown to exert opposite effects on post-ischemic myocardial apoptosis and cardiac function recovery (Yue et al., 2000).

Studies have also shown that the expression of CaMEK is sufficient to activate ERK1/2. Activated ERKs then translocate into the nucleus, where they phosphorylate and activate nuclear transcription factors such as Elk-1, c-fos, and c-jun (Garrington and Johnson, 1999), ultimately reducing reperfusion injury-induced apoptosis and promoting cell survival. Such proliferative effects of CaMEK in confluent IEC-6 cells have been previously reported, and silencing MEK expression significantly reduces the extent of ERK1/2 phosphorylation, inhibiting cell proliferation. These characteristics make a method in which mediated CaMEK efficiently transfects myocardial cells and activates ERK1/2 highly desirable.

For this purpose, we transfected myocardial cells with an AAV9 vector expressing eGFP-tagged CaMEK. High levels of MEK and p-ERK were detected in CaMEK-transfected cells. We then measured MEK and p-ERK protein expression in CaMEK-transfected cells after treatment with PD98059. MEK and p-ERK expression was significant suppressed, indicating that AAV9-mediated CaMEK gene transfer can effectively activate the ERK1/2 pathway. In a static state, ERK is located in the cytoplasm and translocates to the nucleus when activated (Aliaga et al., 1999; Mebratu and Tesfaigzi, 2009). To confirm our results further, we also demonstrated morphologically that ERK1/2 located in the nucleus in the CaMEKtransfected group, whereas minimal nuclear expression occurred in the control group. These results are consistent with the findings of others. In summary, we report that the activation of ERK1/2 by AAV9-eGFP-CaMEK is a novel method that contributes to cardioprotective and anti-apoptotic effects in I/R-injured cells.

\section{ACKNOWLEDGMENTS}

Research supported by a grant from the National Natural Science Foundation of China (\#30760263) and the Special preliminary study of the "973" (\#2010CB535013).

\section{REFERENCES}

Aliaga JC, Deschenes C, Beaulieu JF, Calvo EL, et al. (1999). Requirement of the MAP kinase cascade for cell cycle progression and differentiation of human intestinal cells. Am. J. Physiol. 277: G631-G641.

Bueno OF and Molkentin JD (2002). Involvement of extracellular signal-regulated kinases 1/2 in cardiac hypertrophy and cell death. Circ. Res. 91: 776-781.

Cho HS, Chang SH, Chung YS, Shin JY, et al. (2009). Synergistic effect of ERK inhibition on tetrandrine-induced apoptosis in A549 human lung carcinoma cells. J. Vet. Sci. 10: 23-28.

Garrington TP and Johnson GL (1999). Organization and regulation of mitogen-activated protein kinase signaling pathways. Curr. Opin. Cell Biol. 11: 211-218.

Hajjar RJ and Samulski RJ (2006). Heart failure: a silver bullet to treat heart failure. Gene Ther. 13: 997.

Hausenloy DJ, Tsang A and Yellon DM (2005). The reperfusion injury salvage kinase pathway: a common target for both ischemic preconditioning and postconditioning. Trends Cardiovasc. Med. 15: 69-75.

Kuno Y, Kondo K, Iwata H, Senga T, et al. (1998). Tumor-specific activation of mitogen-activated protein kinase in human colorectal and gastric carcinoma tissues. Jpn. J. Cancer Res. 89: 903-909.

Lemieux E, Bergeron S, Durand V, Asselin C, et al. (2009). Constitutively active MEK1 is sufficient to induce epithelialto-mesenchymal transition in intestinal epithelial cells and to promote tumor invasion and metastasis. Int. J. Cancer 125: $1575-1586$. 
Li DY, Tao L, Liu H, Christopher TA, et al. (2006). Role of ERK1/2 in the anti-apoptotic and cardioprotective effects of nitric oxide after myocardial ischemia and reperfusion. Apoptosis 11: 923-930.

Li XM, Ma YT, Yang YN, Liu F, et al. (2009). Downregulation of survival signalling pathways and increased apoptosis in the transition of pressure overload-induced cardiac hypertrophy to heart failure. Clin. Exp. Pharmacol. Physiol. 36: 1054-1061.

Licato LL and Brenner DA (1998). Analysis of signaling protein kinases in human colon or colorectal carcinomas. Dig. Dis. Sci. 43: 1454-1464.

Lips DJ, Bueno OF, Wilkins BJ, Purcell NH, et al. (2004). MEK1-ERK2 signaling pathway protects myocardium from ischemic injury in vivo. Circulation 109: 1938-1941.

Manning G, Whyte DB, Martinez R, Hunter T, et al. (2002). The protein kinase complement of the human genome. Science 298: 1912-1934.

Mebratu Y and Tesfaigzi Y (2009). How ERK1/2 activation controls cell proliferation and cell death: Is subcellular localization the answer? Cell Cycle 8: 1168-1175.

Pacak CA, Mah CS, Thattaliyath BD, Conlon TJ, et al. (2006). Recombinant adeno-associated virus serotype 9 leads to preferential cardiac transduction in vivo. Circ. Res. 99: e3-e9.

Prasad KM, Xu Y, Yang Z, Acton ST, et al. (2011). Robust cardiomyocyte-specific gene expression following systemic injection of AAV: in vivo gene delivery follows a Poisson distribution. Gene Ther. 18: 43-52.

Simpson P and Savion S (1982). Differentiation of rat myocytes in single cell cultures with and without proliferating nonmyocardial cells. Cross-striations, ultrastructure, and chronotropic response to isoproterenol. Circ. Res. 50: 101116.

Treisman R (1996). Regulation of transcription by MAP kinase cascades. Curr. Opin. Cell Biol. 8: 205-215.

Voisin L, Julien C, Duhamel S, Gopalbhai K, et al. (2008). Activation of MEK1 or MEK2 isoform is sufficient to fully transform intestinal epithelial cells and induce the formation of metastatic tumors. BMC Cancer 8: 337.

White JD, Thesier DM, Swain JB, Katz MG, et al. (2011). Myocardial gene delivery using molecular cardiac surgery with recombinant adeno-associated virus vectors in vivo. Gene Ther. 18: 546-552.

Yue TL, Wang C, Gu JL, Ma XL, et al. (2000). Inhibition of extracellular signal-regulated kinase enhances Ischemia/ Reoxygenation-induced apoptosis in cultured cardiac myocytes and exaggerates reperfusion injury in isolated perfused heart. Circ. Res. 86: 692-699. 